\title{
Consumo de água e eficiência produtiva de plantas de trigo tratadas com Etil-trinexapac
}

\author{
Water consumption and yield efficiency of wheat plants treated with Trinexapac-ethyl
}

\author{
Thais Lemos Turek², Luiz Henrique Michelon ${ }^{1}$, Cláudia Tochetto ${ }^{1}$, Antônio Eduardo Coelho² \& Samuel \\ Luiz Fioreze ${ }^{1^{*}}$
}

${ }^{1}$ Universidade Federal de Santa Catarina, Curitibanos, SC, Brasil. *Autor para correspondência: s.fioreze@ufsc.br.

${ }^{2}$ Universidade do Estado de Santa Catarina, Lages, SC, Brasil.

Submissão: 29/05/2017 / Aceite: 09/05/2018

\begin{abstract}
RESUMO
O uso eficiente de recursos do ambiente de cultivo, o qual é influenciado por características da planta, é de grande importância para a adaptação de culturas em sistemas de produção. O presente trabalho teve como objetivo estudar o consumo de água e a eficiência produtiva de plantas de trigo tratadas com Etiltrinexapac. Foram conduzidos dois experimentos, em cultivo protegido, entre 2014 e 2015 . Utilizou-se o delineamento experimental de blocos casualizados, em esquema fatorial $2 \times 3$, com quatro repetições. Dois cultivares de trigo, foram submetidos à aplicação de três doses de Etil-trinexapac $\left(0,125\right.$ e $188 \mathrm{~g} \mathrm{ha}^{-1}$ de i.a.) na fase de alongamento do colmo principal. Foram avaliados: consumo de água, altura da planta e distância fonte e dreno, e os parâmetros morfológicos da folha bandeira. Ao final do ciclo da cultura, foram avaliados componentes de produção, produção de grãos e índice de colheita da cultura. A aplicação de Etil-trinexapac reduziu o crescimento de plantas de trigo, sem afetar a produção de grãos. A aplicação do regulador não reduz o consumo de água, tampouco o índice de colheita da cultura do trigo.
\end{abstract}

PALAVRAS-CHAVE: Triticum aestivum L., regulador vegetal, arquitetura de planta, eficiência do uso da água, índice de colheita.

\begin{abstract}
Efficient use of the resources of the growing environment, which is affected by plant traits, is very important for crop adaptation on production system. This work aimed to study the water consumption and yield efficiency of wheat plants treated with Trinexapac-ethyl. Two experiments were carried out, in greenhouse conditions, between 2014 and 2015. Experiments were arranged in randomized blocks design in a factorial $2 \times 3$, with four replicates. Two wheat cultivars were sprayed with three Trinexapac-ethyl doses $\left(0,125\right.$ and $188 \mathrm{~g}$ a.i. ha $\left.{ }^{-1}\right)$, at main culm elongation stage. The water consumption, plant height, sourcesink distance and the morphological parameters of the flag leaf were evaluated. At the end of the growing cycle, yield and yield components were determined, as well as harvest index. Trinexapac-ethyl reduced plant growth, without affecting grain production. Plant growth regulator application does not reduce either water consumption or wheat crop harvest index.
\end{abstract}

KEYWORDS: Triticum aestivum L., plant growth regulators, plant architecture, water use efficiency, harvest index.

\section{INTRODUÇÃO}

Elevados níveis de produção na cultura do trigo estão associados ao bom suprimento de água e nutrientes, recursos que devem ser utilizados de forma eficaz pela planta para potencializar o metabolismo e reduzir perdas. Melhorias na eficiência de uso de recursos, como água e nutrientes, podem estar associadas a características da planta, como arquitetura de raízes e de parte aérea (WANG \& LI 2008, YANG \& HWA 2008, CAl et al. 2016).

A arquitetura de uma planta é determinada pela natureza e pelo arranjo de cada um dos seus componentes como resultado do equilíbrio entre o potencial genético e os fatores limitantes impostos pelo ambiente externo (MAITI \& RODRíGUEZ 2010). Fatores endógenos, como o balanço hormonal e a competição por assimilados entre órgãos da planta, também possuem efeitos sobre a arquitetura de plantas (GUO et al. 2011), seja em resposta ao ambiente ou mesmo a técnicas de cultivo. 
Alterações na arquitetura da planta, como um todo ou mesmo em folhas, resultam em melhorias na partição de nitrogênio e assimilados, além de reduzir o consumo de água, que resulta em aumento no índice de colheita da cultura (GUO et al. 2011). A ocorrência de folhas superiores mais eretas e folhas inferiores arranjadas horizontalmente melhora a interceptação da radiação solar pelo dossel de plantas, o que aumenta seu aproveitamento (LONG et al. 2006). Mudanças na morfologia foliar, em termos de espessura e disposição de células, por sua vez, podem resultar em aumento na eficiência fotossintética por unidade de área ou mesmo no controle das trocas gasosas, com efeito direto sobre a fotossíntese e o consumo de água (RICHARDS 2000, THOLEN et al. 2012, SONG et al. 2013)

Reguladores vegetais do grupo dos inibidores da giberelina provocam, entre outros efeitos, a redução do porte (BERTI et al. 2007, ZAGONEL \& FERNANDES 2007, FIOREZE \& RODRIGUES 2014a) e melhorias na arquitetura de plantas (SOUZA \& ROSOLEM 2007, FIALHO et al. 2009, FIOREZE \& RODRIGUES 2014a, CHAVARRIA et al. 2015). A redução na altura de plantas tem como benefícios a redução no acamamento, normalmente associados ao uso de maiores doses de nitrogênio e altas densidades de semeadura (ZAGONEL et al. 2002).

Apesar de as alterações morfológicas proporcionadas pela aplicação de reguladores vegetais em culturas serem bem descritas na literatura, não está claro se as melhorias na arquitetura de plantas, associadas à ação deste tipo de regulador, podem afetar, de alguma forma, o consumo de água pelas plantas e o índice de colheita, por meio de melhorias na partição de assimilados. Neste sentido, o presente trabalho teve como objetivo estudar o consumo de água e o comportamento produtivo de cultivares de trigo tratadas com Etil-trinexapac.

\section{MATERIAL E MÉTODOS}

Foram conduzidos dois experimentos entre os meses de julho e novembro dos anos de 2014 e 2015. Os ensaios foram conduzidos em cultivo protegido na área de pesquisa da Universidade Federal de Santa Catarina, Campus de Curitibanos. A área de cultivo possui controle de umidade e temperatura do ar, a qual foi ajustada para uma faixa 20 e $25{ }^{\circ} \mathrm{C}$ e umidade relativa do ar próxima a $70 \%$ durante a condução dos ensaios.

Utilizou-se o delineamento experimental de blocos casualizados, em esquema fatorial $2 \times 3$, com quatro repetições. O primeiro fator foi composto por dois cultivares de trigo. O segundo fator foi composto por três doses de Etil-trinexapac (0,125 e $188 \mathrm{~g} \mathrm{ha}^{-1}$ de i.a.). No ano de 2014, foram utilizados os cultivares CD 150, com ciclo precoce, porte baixo e médio potencial de perfilhamento, e Quartzo, com ciclo médio e porte e perfilhamento médio. No ano de 2015, utilizou-se os cultivares CD 150 e Pioneiro, com ciclo médio, porte médio/alto e perfilhamento abundante. Cada unidade experimental foi formada por um vaso de polietileno com volume de 10 litros, preenchido com solo corrigido, adubado e cultivado com duas plantas uniformes de trigo.

O solo utilizado foi classificado como Cambissolo Háplico de textura argilosa (EMBRAPA 2006). Antes de serem preenchidos com solo, os vasos foram equipados com sistema de irrigação de subsuperfície, composto por uma cápsula porosa de cerâmica conectada a um reservatório de água por um microtubo flexível transparente. Este sistema é um aprimoramento do modelo proposto por MONTANHEIRO et al. (1979) e modificado por TRAUTMANN et al. (2014), o qual permite a reposição automática de água, conforme a evapotranspiração do vaso, bem como a determinação do consumo de água pelas plantas.

A adubação de base foi realizada com $687 \mathrm{mg} \mathrm{dm}^{-3}$ de fósforo (Super Fosfato Simples), $78 \mathrm{mg} \mathrm{dm}^{-3}$ de potássio $(\mathrm{KCl})$ e $1,5 \mathrm{~g} \mathrm{dm}^{-3}$ de calcário. A semeadura foi realizada de forma manual. Foram semeadas seis sementes por vaso, e mantidas duas plantas uniformes por vaso após desbaste. Para minimizar as perdas de água por evaporação, a superfície do solo dos vasos foi coberta com uma camada uniforme de palha triturada de Urochloa spp.. A adubação nitrogenada de cobertura foi realizada na forma de ureia (45\% de $\mathrm{N}$ ) dissolvida em água, na dose de $25 \mathrm{mg} \mathrm{dm}^{-3}$ de $\mathrm{N}$. Foram realizadas sete aplicações entre a emergência e o período de antese das plantas de trigo, que totalizou $175 \mathrm{mg} \mathrm{dm}^{-3} \mathrm{de} \mathrm{N}$.

A aplicação de Etil-trinexapac foi realizada na fase do primeiro nó visível do colmo principal, que corresponde ao estádio 6 da escala de Feeks (LARGE 1954). Para tanto, foi utilizado o produto comercial Moddus $^{\circledR}$. A aplicação foi realizada via foliar utilizando um pulverizador costal de barras com pressão de $\mathrm{CO}_{2}$ e pontas do tipo leque (110.02) ajustado para um volume de calda de $150 \mathrm{~L} \mathrm{ha}^{-1}$. Para o tratamento testemunha, foi realizada aplicação de água, para uniformizar a superfície de molhamento.

Após a aplicação do regulador, o consumo de água foi monitorado com intervalo de dois dias, em dois períodos, do primeiro ao $14^{\circ}$ dia após a aplicação (DAA), e do 26ํㅡㄹ ao $31^{\circ}$ DAA, período no qual ocorreram a emissão e expansão da folha bandeira das plantas, em que se esperava o efeito do regulador. A 
determinação do consumo de água foi determinada por meio da variação no nível de água no reservatório, descontando-se a quantidade de água evaporada pelo vaso, a qual foi determinada em vasos não cultivados distribuídos entre os blocos.

$\mathrm{Na}$ fase de antese, estádio 10.5 na escala de Feeks (LARGE 1954), foi realizada a retirada total das folhas de uma das plantas de cada vaso. Este procedimento teve como objetivo permitir o estudo da participação dos assimilados acumulados até a antese para o enchimento de grãos. Na fase final do período de enchimento de grãos, foram avaliadas a altura de plantas e a distância entre fonte e dreno. A determinação da distância entre a fonte principal de fotoassimilados (folha bandeira) e o dreno (espiga) foi realizada por meio da medida do comprimento da bainha da folha-bandeira (CB) e da distância entre o nó da folha bandeira e a inserção da espiga (NE). Por meio da soma das duas distâncias obteve-se a distância total de caminhamento de fotoassimilados da folha bandeira até a espiga (FIOREZE \& RODRIGUES 2014a).

Ao final do ciclo da cultura foram determinados os componentes da produção e a produção por planta. Foram determinados o número total de perfilhos e o número de perfilhos férteis (com espigas produtivas), o comprimento da ráquis, o número de espiguetas por espiga, o número de grãos por espigueta e o número e a massa de grãos por espiga. Em seguida, foi determinada a massa de grãos por planta e 0 peso de mil grãos, corrigindo-se a umidade para 13\%. Foi determinado ainda, o índice de colheita, dividindo-se a massa de grãos produzidos por planta pela massa de matéria seca total da planta no momento da colheita.

Os dados foram submetidos à análise de variância pelo teste $F(p<0,05)$. Quando detectadas variações significativas, as médias foram contrastadas pelo teste $t(p<0,05)$. Para o estudo da participação dos assimilados acumulados até a antese para o enchimento de grãos, considerou-se a retirada das folhas como um terceiro fator, obtendo-se um tratamento com e outro sem a retirada das folhas, resultando em um esquema fatorial $2 \times 3 \times 2$.

\section{RESULTADOS E DISCUSSÃO}

No ano de 2014, diferenças no consumo de água foram observadas apenas para os cultivares, aos 27 e 29 DAA de Etil-trinexapac, bem como para o consumo médio e total (Tabela 1). Para estas avaliações, o cultivar Quartzo apresentou consumo de água superior ao CD 150. O efeito das doses de Etil-trinexapac foi observado apenas aos 29 DAA, com menor consumo observado para a aplicação de $188 \mathrm{~g} \mathrm{ha}^{-1}$ do produto, sem diferenças entre os demais tratamentos. O consumo médio e total de água no período não foi afetado pela aplicação do regulador.

A interação entre cultivares e doses de Etil-trinexapac para o consumo de água foi observada apenas aos 31 DAA (Tabela 1). Para o cultivar CD 150, o menor consumo de água foi observado para dose de 188 $\mathrm{g} \mathrm{ha}^{-1}$ (Tabela 2), sem diferir da testemunha sem aplicação. No cultivar Quartzo, contudo, não houve efeito da aplicação de Etil-trinexapac sobre o consumo de água. O cultivar Quartzo apresentou consumo de água superior ao CD 150 apenas no tratamento testemunha, sem haver diferenças entre os mesmos para as demais doses.

No ano de 2015, foram observadas diferenças entre os cultivares no consumo de água aos $5,7,9,11$ e 13 DAA, além do consumo médio e total (Tabela 1), sendo que o cultivar Pioneiro apresentou consumo de água superior ao CD 150, o que pode estar associado ao maior número de perfilhos, característico do cultivar. Entretanto, no segundo período de avaliação, não houve diferença no consumo de água. No período descrito $(5,7,9,11$ e $13 \mathrm{DAA})$, a interação entre os fatores de estudo não foi observada para o ano de 2015.

A eficiência no uso de água (EUA) é de grande importância para a adaptação de culturas em condições de baixa disponibilidade hídrica, sendo que o rendimento da cultura é proporcional ao volume de água transpirada durante o ciclo multiplicado pela eficiência no uso de água e pelo índice de colheita (PIMENTEL 2004). Foi possível observar que a cultivar CD 150, nos dois anos de cultivo, apresentou média de consumo de água inferior aos demais cultivares. Analisando estes resultados juntamente com a produção e o índice de colheita para os dois anos (Tabela 3), observa-se provável maior EUA para CD 150, haja vista que não houve diferenças de produção e índice de colheita. Este comportamento, contudo, precisa ainda ser melhor estudado.

Resultados referentes ao consumo de água em plantas tratadas com reguladores de crescimento são escassos na literatura. É conhecido que a aplicação de Etil-trinexapac provoca redução da altura de plantas e melhorias na arquitetura da parte aérea (BERTI et al. 2007, ZAGONEL \& FERNANDES 2007, FIOREZE \& RODRIGUES 2014a). FIALHO et al. (2009) observaram que plantas de Urochloa brizantha apresentaram 
redução no tamanho de folhas, associado ao espessamento do limbo foliar, como reflexo do aumento do mesofilo e bainha foliar. CHAVARRIA et al. (2015) observaram redução de até $16,54 \%$ na área foliar de plantas de dois cultivares de trigo tratadas com Etil-trinexapac.

Tabela 1. Consumo de água em cultivares de trigo (C) tratados com Etil-trinexapac (E) em cultivo protegido. Curitibanos, SC, safras 2014 e 2015.

Table 1. Water consumption in wheat cultivars (C) treated with Trinexapac-ethyl $(E)$ in greenhouse conditions. Curitibanos, SC, 2014 and 2015 growing seasons.

\begin{tabular}{|c|c|c|c|c|c|c|c|}
\hline \multicolumn{8}{|c|}{$2014\left(\mathrm{ml} \mathrm{vaso}^{-1}\right)$} \\
\hline $\mathrm{FV}^{1}$ & $1 \mathrm{DAA}^{2}$ & 3 DAA & 5 DAA & 7 DAA & $9 \mathrm{DAA}$ & 11 DAA & $13 \mathrm{DAA}$ \\
\hline CD 150 & 135,79 & 193,53 & 174,58 & 144,64 & 158,42 & 316,00 & 197,92 \\
\hline Quartzo & 149,89 & 213,11 & 190,75 & 164,00 & 227,97 & 287,31 & 192,22 \\
\hline$p^{*}(C)$ & 0,36 & 0,34 & 0,29 & 0,19 & 0,12 & 0,35 & 0,85 \\
\hline $0 \mathrm{~g} \mathrm{ha}^{-1}$ & 145,34 & 201,25 & 180,50 & 149,38 & 232,63 & 280,25 & 206,50 \\
\hline $125 \mathrm{~g} \mathrm{ha}^{-1}$ & 147,71 & 214,17 & 196,88 & 166,75 & 186,46 & 312,63 & 193,71 \\
\hline $188 \mathrm{~g} \mathrm{ha}^{-1}$ & 135,48 & 194,54 & 170,63 & 146,83 & 160,50 & 312,08 & 185,00 \\
\hline $\mathrm{p}(\mathrm{E})$ & 0,78 & 0,72 & 0,37 & 0,48 & 0,39 & 0,61 & 0,83 \\
\hline$p(C \times E)^{3}$ & 0,13 & 0,76 & 0,58 & 0,63 & 0,28 & 0,69 & 0,84 \\
\hline CV $(\%)^{4}$ & 25,90 & 24,00 & 19,80 & 22,60 & 53,20 & 24,30 & 36,20 \\
\hline $\mathrm{FV}$ & Médio $^{5}$ & Total $^{6}$ & 27 DAA & 29 DAA & $31 \mathrm{DAA}$ & Médio $^{7}$ & Total $^{8}$ \\
\hline CD 150 & 188,70 & 1320,88 & $319,92 \mathrm{~b}$ & $416,11 b$ & 500,67 & $412,23 \mathrm{~b}$ & $1236,69 \mathrm{~b}$ \\
\hline Quartzo & 203,61 & 1425,25 & $410,42 \mathrm{a}$ & $512,84 \mathrm{a}$ & 590,23 & $504,50 \mathrm{a}$ & $1513,49 a$ \\
\hline $\mathrm{p}(\mathrm{C})$ & 0,18 & 0,18 & 0,01 & 0,04 & 0,12 & 0,03 & 0,03 \\
\hline $0 \mathrm{~g} \mathrm{ha}^{-1}$ & 199,41 & 1395,84 & 369,13 & $459,38 a b$ & 537,50 & 455,33 & 1366,00 \\
\hline $125 \mathrm{~g} \mathrm{ha}^{-1}$ & 202,61 & 1418,29 & 400,75 & $543,89 \mathrm{~b}$ & 600,97 & 515,20 & 1545,61 \\
\hline $188 \mathrm{~g} \mathrm{ha}^{-1}$ & 186,44 & 1305,06 & 325,63 & 390,17 a & 497,88 & 404,56 & 1213,67 \\
\hline $\mathrm{p}(\mathrm{E})$ & 0,44 & 0,44 & 0,17 & 0,04 & 0,32 & 0,10 & 0,10 \\
\hline $\mathrm{p}(\mathrm{C} \times \mathrm{E})$ & 0,48 & 0,48 & 0,14 & 0,16 & 0,04 & 0,06 & 0,06 \\
\hline $\mathrm{CV}(\%)$ & 13,30 & 13,30 & 20,50 & 23,00 & 24,40 & 21,10 & 21,10 \\
\hline \multicolumn{8}{|c|}{$2015\left(\mathrm{ml} \mathrm{vaso}^{-1}\right)$} \\
\hline FV & $1 \mathrm{DAA}$ & $3 \mathrm{DAA}$ & 5 DAA & 7 DAA & $9 \mathrm{DAA}$ & $11 \mathrm{DAA}$ & 13 DAA \\
\hline CD 150 & 30,25 & 75,81 & $60,00 \mathrm{~b}$ & $73,33 \mathrm{~b}$ & $77,92 \mathrm{~b}$ & $54,25 \mathrm{~b}$ & $76,42 \mathrm{~b}$ \\
\hline Pioneiro & 46,58 & 90,92 & $190,67 \mathrm{a}$ & 304,33 a & $216,83 \mathrm{a}$ & $105,50 \mathrm{a}$ & $242,92 \mathrm{a}$ \\
\hline $\mathrm{p}(\mathrm{C})$ & 0,16 & 0,44 & 0,01 & 0,00 & 0,00 & 0,03 & 0,00 \\
\hline $0 \mathrm{~g} \mathrm{ha}^{-1}$ & 38,88 & 83,38 & 112,75 & 182,25 & 153,75 & 76,25 & 153,00 \\
\hline $125 \mathrm{~g} \mathrm{ha}^{-1}$ & 39,88 & 82,33 & 133,25 & 185,88 & 145,00 & 82,63 & 162,75 \\
\hline $188 \mathrm{~g} \mathrm{ha}^{-1}$ & 36,50 & 84,38 & 130,00 & 198,38 & 143,38 & 80,75 & 163,25 \\
\hline$p(E)$ & 0,97 & 1,00 & 0,90 & 0,97 & 0,96 & 0,97 & 0,97 \\
\hline$p(C \times E)$ & 0,88 & 0,43 & 0,76 & 0,82 & 0,68 & 0,81 & 0,86 \\
\hline $\mathrm{CV}(\%)$ & 70,40 & 56,30 & 77,20 & 70,50 & 54,30 & 63,90 & 55,40 \\
\hline FV & Médio & Total & 27DAA & 29DAA & 31DAA & Médio & Total \\
\hline CD 150 & $64,00 \mathrm{~b}$ & $447,97 \mathrm{~b}$ & 155,17 & 182,75 & 136,58 & 158,17 & 474,50 \\
\hline Pioneiro & $171,11 \mathrm{a}$ & $1197,75 \mathrm{a}$ & 155,83 & 234,75 & 166,00 & 185,53 & 556,58 \\
\hline$p(C)$ & 0,00 & 0,00 & 0,98 & 0,25 & 0,37 & 0,44 & 0,44 \\
\hline $0 \mathrm{~g} \mathrm{ha}^{-1}$ & 114,32 & 800,25 & 162,00 & 201,75 & 151,63 & 171,79 & 515,38 \\
\hline $125 \mathrm{~g} \mathrm{ha}^{-1}$ & 118,82 & 831,71 & 160,50 & 220,50 & 161,75 & 180,92 & 542,75 \\
\hline $188 \mathrm{~g} \mathrm{ha}^{-1}$ & 119,52 & 836,63 & 144,00 & 204,00 & 140,50 & 162,83 & 488,50 \\
\hline $\mathrm{p}(\mathrm{E})$ & 0,99 & 0,99 & 0,86 & 0,93 & 0,86 & 0,91 & 0,91 \\
\hline$p(C \times E)$ & 0,76 & 0,76 & 0,80 & 0,77 & 0,71 & 0,76 & 0,76 \\
\hline CV (\%) & 61,60 & 61,60 & 45,90 & 50,60 & 51,80 & 49,00 & 49,00 \\
\hline
\end{tabular}

${ }^{1}$ Fonte de variação; ${ }^{2}$ Dias após a aplicação de Etil-trinexapac; ${ }^{3}$ Cultivar X Etil-trinexapac, ${ }^{4}$ Coeficiente de Variação; $;$ Consumo médio entre 1 e $13 \mathrm{DAA} ;{ }^{6}$ Consumo total entre 1 e $13 \mathrm{DAA} ;{ }^{7}$ Consumo médio entre 27 e 31 DAA; ${ }^{8}$ Consumo total entre 27 e 31 DAA; ${ }^{*}$ Valor da probabilidade do Teste $F$. Médias de mesma letra não diferem entre si pelo teste $T(p<0,05)$.

Tabela 2. Efeito da interação entre cultivares de trigo e aplicação de Etil-trinexapac sobre o consumo de água de plantas aos 31 dias após a aplicação. Curitibanos, SC, safra 2014.

Table 2. Interaction effect between wheat cultivars and Trinexapac-ethyl application for water plant consumption at 31 days after application. Curitibanos, SC, 2014 growing season.

\begin{tabular}{rlc}
\hline \multicolumn{1}{l}{ Doses } & \multicolumn{2}{c}{ Consumo de água $(\mathrm{mL}$ vaso-1) } \\
& CD 150 & Quartzo \\
\hline $0 \mathrm{~g} \mathrm{ha}^{-1}$ & $429,50 \mathrm{Aab}$ & $645,50 \mathrm{Ba}$ \\
$125 \mathrm{~g} \mathrm{ha}^{-1}$ & $665,70 \mathrm{Ab}$ & $536,20 \mathrm{Aa}$ \\
$188 \mathrm{~g} \mathrm{ha}^{-1}$ & $406,80 \mathrm{Aa}$ & $589,00 \mathrm{Aa}$ \\
\hline
\end{tabular}

Médias seguidas de mesma letra, maiúscula na horizontal e minúscula na vertical não diferem entre si; pelo teste T ( $p<0,05 \%)$. 
FIOREZE \& RODRIGUES (2014a) observaram redução de área foliar em plantas de trigo tratadas com Etil-trinexapac, contudo, a taxa transpiratória das plantas não foi afetada. Se a taxa de perda de água por unidade de área foliar é constante, a redução na perda de água por plantas pode ocorrer em função da redução de área foliar. ALVAREZ et al. (2016) observaram aumento pontual na assimilação líquida de $\mathrm{CO}_{2}$, transpiração e condutância estomática em plantas de arroz tratadas com Etil-trinexapac, sem ocorrer redução de área foliar. É possível, portanto, que o menor consumo de água em plantas de trigo tratadas com Etil-trinexapac esteja associado à redução da área foliar das plantas.

No ano de 2014, observou-se o efeito das doses de Etil-trinexapac sobre a altura de plantas, o comprimento do último entrenó do colmo principal, o comprimento da bainha da folha bandeira e a distância entre fonte e dreno (Tabela 4). Menores valores de altura, comprimento da bainha e distância fonte-dreno foram observadas para a dose de $188 \mathrm{~g} \mathrm{ha}^{-1}$, sendo que a dose de $125 \mathrm{~g} \mathrm{ha}^{-1}$ não diferiu da testemunha sem aplicação. Para os parâmetros relacionados à altura de plantas e à distância fonte-dreno, não houve diferença entre os cultivares, tampouco a interação entre os fatores.

Tabela 3. Componentes da produção e produção de grãos em cultivares de trigo (C) tratadas com Etiltrinexapac $(E)$ e submetidas à desfolha $(D)$ no período de antese. Curitibanos, SC, safras $2014 \mathrm{e}$ 2015.

Table 3. Yield and yield components in wheat cultivars (C) treated with Trinexapac-ethyl (E) and affected by defoliation (D) at anthesis. Curitibanos, SC, 2014 and 2015 growing seasons.

\begin{tabular}{|c|c|c|c|c|c|c|c|c|c|}
\hline \multicolumn{10}{|c|}{2014} \\
\hline $\mathrm{FV}^{1}$ & NESP & $\mathrm{CR}(\mathrm{cm})$ & NESPG & NGESP & NGESPG & MGESP (g) & MGP $(g)$ & MMG (g) & IC \\
\hline CD 150 & 14,04 & $8,73 \mathrm{a}$ & 18,92 & 42,53 & 2,25 & 1,43 & 19,69 & 33,42 & 0,35 \\
\hline Quartzo & 13,54 & $7,88 \mathrm{~b}$ & 18,81 & 42,14 & 2,24 & 1,43 & 19,18 & 33,67 & 0,35 \\
\hline $\mathrm{p}(\mathrm{C})$ & 0,54 & 0,00 & 0,61 & 0,77 & 0,89 & 0,86 & 0,65 & 0,69 & 0,67 \\
\hline $0 \mathrm{~g} \mathrm{ha}^{-1}$ & 12,75 & 8,49 & 18,90 & 44,64 & $2,36 \mathrm{~b}$ & $1,57 \mathrm{~b}$ & 19,66 & $35,19 \mathrm{~b}$ & 0,33 \\
\hline $125 \mathrm{~g} \mathrm{ha}^{-1}$ & 14,25 & 8,24 & 18,70 & 41,44 & $2,22 \mathrm{ab}$ & $1,41 \mathrm{a}$ & 20,18 & 33,99 b & 0,36 \\
\hline $188 \mathrm{~g} \mathrm{ha}^{-1}$ & 14,38 & 8,18 & 18,99 & 40,92 & $2,16 \mathrm{a}$ & $1,30 \mathrm{a}$ & 18,45 & $31,44 \mathrm{a}$ & 0,36 \\
\hline$p(E)$ & 0,20 & 0,53 & 0,52 & 0,06 & 0,04 & 0,00 & 0,45 & 0,00 & 0,25 \\
\hline Testemunha & 13,92 & 8,34 & 18,89 & 43,46 & 2,30 & $1,63 \mathrm{a}$ & $22,36 \mathrm{a}$ & $37,45 \mathrm{a}$ & 0,35 \\
\hline Desfolha & 13,67 & 8,26 & 18,84 & 41,21 & 2,19 & $1,23 b$ & $16,50 \mathrm{~b}$ & $29,63 \mathrm{~b}$ & 0,35 \\
\hline $\mathrm{p}(\mathrm{D})$ & 0,76 & 0,74 & 0,80 & 0,10 & 0,08 & 0,00 & 0,00 & 0,00 & 0,77 \\
\hline $\mathrm{p}(\mathrm{D} \times \mathrm{C})^{2}$ & 0,68 & 0,77 & 0,84 & 0,09 & 0,07 & 0,05 & 0,67 & 0,54 & 0,95 \\
\hline $\mathrm{p}(\mathrm{DxE})^{3}$ & 0,68 & 0,77 & 0,93 & 0,26 & 0,13 & 0,09 & 0,37 & 0,34 & 0,90 \\
\hline $\mathrm{p}(\mathrm{CxE})^{4}$ & 0,05 & 1,00 & 0,50 & 0,22 & 0,07 & 0,11 & 0,08 & 0,43 & 0,65 \\
\hline $\mathrm{p}(\mathrm{DxC} \times \mathrm{E})^{5}$ & 0,09 & 0,86 & 0,12 & 0,68 & 0,45 & 0,09 & 0,47 & 0,10 & 0,91 \\
\hline $\operatorname{CV}(\%)^{6}$ & 20,40 & 9,90 & 3,80 & 10,90 & 9,70 & 11,70 & 20,20 & 6,40 & 18,26 \\
\hline \multicolumn{10}{|c|}{2015} \\
\hline FV & NESP & $\mathrm{CR}(\mathrm{cm})$ & NESPG & NGESP & NGESPG & MGESP (g) & MGP (g) & MMG (g) & IC \\
\hline CD 150 & $6,04 \mathrm{~b}$ & $7,10 \mathrm{~b}$ & 18,00 & $34,60 \mathrm{~b}$ & $1,90 \mathrm{~b}$ & 1,10 & $6,50 \mathrm{~b}$ & 29,84 & 0,32 \\
\hline Pioneiro & $8,42 \mathrm{a}$ & $8,60 \mathrm{a}$ & 18,20 & $38,90 \mathrm{a}$ & $2,10 a$ & 1,20 & $9,80 \mathrm{a}$ & 29,90 & 0,32 \\
\hline $\mathrm{p}(\mathrm{C})$ & 0,00 & 0,00 & 0,50 & 0,03 & 0,01 & 0,10 & 0,00 & 0,96 & 0,81 \\
\hline $0 \mathrm{~g} \mathrm{ha}^{-1}$ & 6,87 & 8,00 & 18,10 & $40,10 \mathrm{~b}$ & $2,20 \mathrm{~b}$ & $1,30 \mathrm{~b}$ & 8,90 & $32,35 \mathrm{~b}$ & 0,32 \\
\hline $125 \mathrm{~g} \mathrm{ha}^{-1}$ & 7,50 & 8,00 & 18,40 & $36,20 a b$ & $2,00 \mathrm{a}$ & $1,10 \mathrm{a}$ & 8,10 & $29,10 \mathrm{a}$ & 0,32 \\
\hline $188 \mathrm{~g} \mathrm{ha}^{-1}$ & 7,31 & 7,60 & 17,90 & $34,00 \mathrm{a}$ & $1,90 \mathrm{a}$ & $1,00 \mathrm{a}$ & 7,30 & $28,16 \mathrm{a}$ & 0,32 \\
\hline$p(E)$ & 0,72 & 0,13 & 0,27 & 0,02 & 0,01 & 0,00 & 0,30 & 0,01 & 0,77 \\
\hline Testemunha & 7,54 & 7,70 & 17,90 & $38,60 \mathrm{a}$ & $2,20 a$ & $1,30 \mathrm{a}$ & $10,10 \mathrm{a}$ & $34,46 \mathrm{a}$ & $0,33 a$ \\
\hline Desfolha & 6,92 & 8,00 & 18,30 & $35,00 \mathrm{~b}$ & $1,90 \mathrm{~b}$ & $0,90 \mathrm{~b}$ & $6,10 \mathrm{~b}$ & 25,27 b & $0,30 \mathrm{~b}$ \\
\hline $\mathrm{p}(\mathrm{D})$ & 0,34 & 0,69 & 0,18 & 0,01 & 0,03 & 0,00 & 0,00 & 0,00 & 0,00 \\
\hline$p(D x C)^{2}$ & 0,34 & 0,13 & 0,12 & 0,64 & 0,97 & 0,76 & 0,85 & 0,62 & 0,67 \\
\hline$p(D x E)^{3}$ & 0,74 & 0,74 & 0,54 & 0,54 & 0,31 & 0,35 & 0,98 & 0,30 & 0,64 \\
\hline$p(\mathrm{C} x \mathrm{E})^{4}$ & 0,89 & 0,93 & 0,33 & 0,50 & 0,28 & 0,05 & 0,33 & 0,05 & 0,40 \\
\hline$p(D x C x E)^{5}$ & 0,85 & 0,32 & 0,45 & 0,20 & 0,16 & 0,53 & 0,69 & 0,58 & 0,72 \\
\hline $\mathrm{CV}(\%)$ & 31,09 & 7,39 & 5,03 & 15,61 & 15,09 & 21,39 & 34,48 & 13,16 & 9,45 \\
\hline
\end{tabular}

NESP: número de espigas por planta; CR: comprimento da ráquis (cm); NESPG: número de espiguetas por espiga; NGESP: número de grãos por espiga; NGESPG: número de grãos por espigueta; MGESP: massa de grãos por espiga (g); MGP: massa de grãos por planta (g); MMG: massa de mil grãos (g); IC: índice de colheita; ${ }^{1}$ Fonte de variação; ${ }^{2}$ Desfolha $x$ Cultivar; ${ }^{3}$ Desfolha $x$ Etil-trinexapac; ${ }^{4}$ Cultivar x Etil-trinexapac; ${ }^{5}$ Desfolha x Cultivar x Etil-trinexapac; ${ }^{6}$ Coeficiente de variação; Valor de probabilidade do Teste F. Médias seguidas de mesma letra não diferem entre si pelo teste $T(p<0,05 \%)$.

No ano de 2015, houve diferença entre cultivares para altura, comprimento da bainha da folha bandeira, comprimento da folha bandeira e largura da folha bandeira (Tabela 4). Para altura e comprimento da bainha da folha bandeira, os menores valores foram observados no cultivar CD 150, enquanto para comprimento da folha bandeira e largura da folha bandeira, os menores valores foram observados no 
cultivar Pioneiro. Houve interação entre cultivares e as doses do regulador para altura, comprimento do último entrenó do colmo principal e distância fonte e dreno.

Tabela 4. Altura de plantas (ALT), comprimento do último entrenó do colmo principal (CE), comprimento da bainha da folha bandeira (CB), distância fonte e dreno (DFD), comprimento folha bandeira (CFB) e largura folha bandeira (LFB) de cultivares de trigo (C) tratados com Etil-trinexapac (E), em cultivo protegido. Curitibanos, SC, safras 2014 e 2015.

Table 4. Plant height (ALT), upper internode of mean culm length (CE), flag leaf sheath length $(C B)$, sourcesink distance (DFD), flag leaf length (CFB) and width (LFB) in wheat cultivars (C) treated with Trinexapac-ethyl (E) in greenhouse conditions. Curitibanos, SC, 2014 and 2015 growing seasons.

\begin{tabular}{|c|c|c|c|c|c|c|}
\hline \multicolumn{7}{|c|}{2014} \\
\hline $\mathrm{FV}^{1}$ & $\mathrm{ALT}(\mathrm{cm})$ & $\mathrm{CE}(\mathrm{cm})$ & $\mathrm{CB}(\mathrm{cm})$ & DFD (cm) & CFB (cm) & LFB (cm) \\
\hline CD 150 & 72,46 & 28,16 & 16,07 & 44,23 & 14,99 & 1,52 \\
\hline Quartzo & 70,72 & 30,42 & 15,57 & 45,99 & 15,06 & 1,45 \\
\hline$p^{*}(C)$ & 0,59 & 0,07 & 0,43 & 0,28 & 0,98 & 0,24 \\
\hline $0 \mathrm{~g} \mathrm{ha}^{-1}$ & $77,53 \mathrm{~b}$ & $32,47 b$ & $16,80 \mathrm{~b}$ & $49,26 \mathrm{~b}$ & 15,30 & 1,43 \\
\hline $125 \mathrm{~g} \mathrm{ha}^{-1}$ & $74,18 \mathrm{~b}$ & $30,07 \mathrm{~b}$ & $16,07 \mathrm{ab}$ & $46,14 b$ & 15,56 & 1,51 \\
\hline $188 \mathrm{~g} \mathrm{ha}^{-1}$ & 63,07 a & $25,34 \mathrm{a}$ & $14,59 \mathrm{a}$ & 39,93 a & 14,22 & 1,50 \\
\hline$p(E)$ & 0,01 & 0,00 & 0,03 & 0,00 & 0,79 & 0,46 \\
\hline$p(C \times E)^{2}$ & 0,14 & 0,06 & 0,61 & 0,12 & 0,39 & 0,50 \\
\hline $\mathrm{CV}(\%)^{3}$ & 10,74 & 9,68 & 9,49 & 8,56 & 27,63 & 10,07 \\
\hline \multicolumn{7}{|c|}{2015} \\
\hline $\mathrm{FV}$ & ALT $(\mathrm{cm})$ & $\mathrm{CE}(\mathrm{cm})$ & CB (cm) & DFD (cm) & CFB (cm) & LFB (cm) \\
\hline CD 150 & $60,93 \mathrm{~b}$ & 26,12 & $15,71 \mathrm{~b}$ & 41,58 & $25,63 \mathrm{a}$ & $1,44 \mathrm{a}$ \\
\hline Pioneiro & $69,03 \mathrm{a}$ & 24,17 & $19,62 \mathrm{a}$ & 43,81 & $18,08 \mathrm{~b}$ & $1,20 \mathrm{~b}$ \\
\hline $\mathrm{p}(\mathrm{C})$ & 0,00 & 0,14 & 0,00 & 0,23 & 0,00 & 0,00 \\
\hline $0 \mathrm{~g} \mathrm{ha}^{-1}$ & 79,84 & 32,69 & 16,88 & 49,19 & 20,43 & 1,26 \\
\hline $125 \mathrm{~g} \mathrm{ha}^{-1}$ & 63,85 & 23,55 & 16,86 & 40,45 & 23,80 & 1,34 \\
\hline $188 \mathrm{~g} \mathrm{ha}^{-1}$ & 51,25 & 19,19 & 19,25 & 38,44 & 21,34 & 1,36 \\
\hline$p(E)$ & 0,00 & 0,00 & 0,16 & 0,00 & 0,27 & 0,54 \\
\hline$p(C \times E)$ & 0,00 & 0,00 & 0,06 & 0,00 & 0,07 & 0,54 \\
\hline $\mathrm{CV}(\%)$ & 6,66 & 12,19 & 15,43 & 10,18 & 18,90 & 13,84 \\
\hline
\end{tabular}

${ }^{1}$ Fonte de variação; ${ }^{2}$ Cultivar x Etil-trinexapac; ${ }^{3}$ Coeficiente de variação; ${ }^{*}$ Valor de probabilidade do Teste F. Médias seguidas de mesma letra não diferem entre si.

No cultivar CD 150, a dose de $188 \mathrm{~g} \mathrm{ha}^{-1}$ resultou na menor altura de plantas, sendo que a dose de $125 \mathrm{~g} \mathrm{ha}^{-1}$ apresentou valores intermediários, menores do que a testemunha (Tabela 5). Para o Pioneiro, as duas doses aplicadas apresentaram redução de altura de plantas em comparação com a testemunha, sem diferirem entre si. O cultivar CD 150 apresentou no comprimento do último entrenó do colmo principal para dose de $188 \mathrm{~g} \mathrm{ha}^{-1}$, enquanto, para o cultivar Pioneiro, as duas doses apresentaram efeito sobre a característica quando comparados com a testemunha. A distância fonte e dreno foi reduzida com a dose de $188 \mathrm{~g} \mathrm{ha}^{-1}$ do regulador para o cultivar CD 150, enquanto, para o Pioneiro, houve efeito apenas para a aplicação de $125 \mathrm{~g} \mathrm{ha}^{-1}$.

A redução no crescimento vegetativo de plantas de cultivares de trigo também foi observada por ZAGONEL et al. (2002), BERTI et al. (2007), ZAGONEL \& FERNANDES (2007) e CHAVARRIA et al. (2015), sem haver interação entre cultivares e doses, semelhante ao apresentado no presente estudo. A redução da altura de plantas em resposta à aplicação de Etil-trinexapac esteve associada à redução do comprimento dos entrenós do colmo (Tabela 4), sendo que o regulador também afetou o crescimento da bainha da folha bandeira, o que reduziu, assim, a distância entre fonte e dreno. A redução na distância entre fonte e dreno foi observada também por FIOREZE \& RODRIGUES (2014a) em resposta à aplicação de Etiltrinexapac.

No ano de 2014 houve diferença entre cultivares para comprimento da ráquis da espiga, sendo que a cultivar CD 150 apresentou a maior média (Tabela 3). O efeito da aplicação de Etil-trinexapac foi observado sobre o número de grãos por espigueta, a massa de grãos por espiga e a massa de mil grãos, em que as maiores médias foram da testemunha. Não houve interação entre os fatores.

No ano de 2015, o cultivar Pioneiro apresentou produção de grãos superior à CD 150, em função do maior número de espigas por planta e de grãos por espiga, considerando que os demais componentes não foram afetados (Tabela 3). A aplicação de Etil-trinexapac provocou a redução no número e, por consequência, na massa de grãos por espiga, independentemente da dose aplicada; contudo a produção final da cultura não foi afetada. 
Tabela 5. Efeito da interação entre cultivares de trigo e aplicação de Etil-trinexapac sobre a altura (ALT), comprimento do último entrenó do colmo principal (CE) e distância fonte e dreno (DFD). Curitibanos, SC, safra 2015.

Table 5. Interaction effect between wheat cultivars and Trinexapac-ethyl application for plant heigth (ALT), upper internode of mean culm length (CE) and source-sink distance (DFD). Curitibanos, SC, 2015 growing season.

\begin{tabular}{|c|c|c|c|c|c|c|}
\hline & \multicolumn{2}{|c|}{$\mathrm{ALT}(\mathrm{cm})$} & \multicolumn{2}{|c|}{$\mathrm{CE}(\mathrm{cm})$} & \multicolumn{2}{|c|}{ DFD (cm) } \\
\hline Dose & CD 150 & Pioneiro & CD 150 & Pioneiro & CD 150 & Pioneiro \\
\hline $0 \mathrm{~g} \mathrm{ha}^{-1}$ & $73,50 \mathrm{Ac}$ & $86,18 \mathrm{Bb}$ & $32,28 \mathrm{Ab}$ & $33,00 \mathrm{Ac}$ & $47,88 \mathrm{Ab}$ & $50,50 \mathrm{Ab}$ \\
\hline $125 \mathrm{~g} \mathrm{ha}^{-1}$ & $65,08 \mathrm{Ab}$ & $62,63 \mathrm{Aa}$ & $30,10 \mathrm{Bb}$ & $17,00 \mathrm{Aa}$ & $45,73 \mathrm{Bb}$ & $35,18 \mathrm{Aa}$ \\
\hline $188 \mathrm{~g} \mathrm{ha}^{-1}$ & $44,20 \mathrm{Aa}$ & $58,30 \mathrm{Ba}$ & $15,88 \mathrm{Aa}$ & $22,50 \mathrm{Bb}$ & $31,13 \mathrm{Aa}$ & $45,75 \mathrm{Bb}$ \\
\hline
\end{tabular}

A massa de mil grãos decresceu em função do aumento da dose de Etil-trinexapac, principalmente no ano de 2015. FIOREZE \& RODRIGUES (2014a) observaram que apesar de a aplicação do regulador provocar redução na distância fonte e dreno e no desenvolvimento da folha bandeira, o peso de mil grãos pode ser reduzido, sem haver prejuízo à produtividade. É importante ressaltar que, mesmo provocando redução na altura de plantas (Tabela 4), a aplicação de Etil-trinexapac não afetou o índice de colheita, o que produziu plantas mais compactas, sem alteração nas proporções de massa, haja vista que a produção de grãos não foi afetada (Tabela 3).

A ausência de efeitos da aplicação de Etil-trinexapac sobre a produtividade da cultura do trigo é frequente (FIOREZE \& RODRIGUES 2014a, 2014b), mesmo quando alguns componentes da produção são afetados, o que demonstra uma boa plasticidade produtiva da cultura. Incrementos de produtividade em função do uso do regulador foram observados por CHAVARRIA et al. (2015) e ZAGONEL et al. (2002). LOZANO \& LEADEN (2002) observaram ganhos significativos de produtividade através da aplicação de Etiltrinexapac em cultivares de trigo, os quais apresentaram folhas mais eretas. De acordo com ZAGONEL \& FERNANDES (2007), os efeitos podem variar conforme a cultivar, pois sofrem influência de características genéticas.

A retirada de todas as folhas da planta no período de antese reduziu a produção de grãos nos dois anos de experimento (Tabela 3 ), independentemente do cultivar ou da aplicação de regulador. No primeiro ano, a redução na produção esteve associada apenas à redução da massa de grãos, enquanto, no segundo ano, o número de grãos também foi afetado. Resultados semelhantes foram observados por SOUZA et al. (2013). Um dos fatores mais importantes para o enchimento de grãos é o acúmulo de assimilados na fase de pré-antese, haja vista que após esta etapa os fotoassimilados começam a diminuir devido à senescência foliar, principalmente em condições de estresse (ASSENG \& VAN HERWAARDEN 2003, INOUE et al. 2004). Em virtude dos resultados observados por ALVAREZ et al. (2016), pode-se esperar que plantas tratadas com Etil-trinexapac apresentassem capacidade de manutenção do potencial produtivo em resposta à desfolha, em função de um maior acúmulo de assimilados no colmo até o período da antese. Este resultado, contudo, não foi observado.

\section{CONCLUSÃO}

A aplicação do Etil-trinexapac reduz o porte das plantas de trigo, sem afetar a produção de grãos.

$\mathrm{O}$ uso de Etil-trinexapac, nas doses recomendadas, não afeta o consumo de água e o índice de colheita da cultura do trigo.

\section{REFERÊNCIAS}

ALVAREZ RCF et al. 2016. Trinexapac-ethyl affects growth and gas exchange of upland rice. Revista Caatinga 29: 320326.

ASSENG S \& VAN HERWAARDEN AF. 2003. Analysis of the benefits to wheat yield from assimilates stored prior to grain filling in a range of environments. Plant and Soil 256: 217-229.

BERTI M et al. 2007. Produtividade de cultivares de trigo em função do trinexapac ethyl e doses de nitrogênio. Scientia Agraria 8: 127-134.

CAI G et al. 2016. Genetic dissection of plant architecture and yield-related traits in Brassica napus. Scientific Reports 6: 2-16.

CHAVARRIA G et al. 2015. Regulador de crescimento em plantas de trigo: reflexos sobre o desenvolvimento vegetativo, rendimento e qualidade de grãos. Revista Ceres 62: 583-588.

EMBRAPA. 2006. Empresa Brasileira de Pesquisa Agropecuária. Sistema brasileiro de classificação de solos. 2.ed. Rio de Janeiro: EMBRAPA. 306p. 
FIALHO CMT et al. 2009. Caracteres morfoanatômicos de Brachiaria brizantha submetida à aplicação de trinexapacethyl. Planta Daninha 27: 533-539.

FIOREZE SL \& RODRIGUES JD. 2014a. Componentes produtivos do trigo afetados pela densidade de semeadura e aplicação de regulador vegetal. Semina: Ciências Agrárias 35: 39-53.

FIOREZE SL \& RODRIGUES JD. 2014b. Tillering affected by sowing density and growth regulators in wheat. Semina: Ciências Agrárias 35: 589-604.

GUO Y et al. 2011. Plant growth and architectural modeling and its applications. Annals of Botany 107: 723-727.

INOUE $T$ et al. 2004. Contribution of pre-anthesis assimilates and current photosynthesis to grain yield, and their relationships to drought resistance in wheat cultivars grown under different soil moisture. Photosynthetica 42: 99-104.

LARGE EC. 1954. Growth stages in cereals illustration of the feeks scale. Plant Pathology 3: 128-129.

LONG SP et al. 2006. Can improvement in photosynthesis increase crop yields? Plant, Cell and Environment 29: $315-$ 330.

LOZANO CM \& LEADEN MI. 2002. Efecto de reguladores de crecimiento sobre el rendimiento y altura en dos cultivares de trigo. In: CONGRESSO NACIONAL DE TRIGO, 5., SIMPÓSIO NACIONAL DE CEREALES DE SIEMBRA OTOÑO INVERNAL, 3. Argentina; Facultad de Ciencias Agrarias UNMdP. 3p.

MAITI R \& RODRÍGUEZ HG. 2010. Plant architecture determines the productivity potential of a crop: a biochemical genetics tool. International Journal of Bio-Resource and Stress Management 1: 1-3.

MONTANHEIRO MN et al. 1979. Controle de tensões de água no solo em vasos com feijoeiro (Phaseolus vulgaris). In: CONGRESSO BRASILEIRO DE CIÊNCIA DO SOLO,17, Manaus. Anais... Campinas: SBCS. p. 68.

PIMENTEL C. 2004. A relação da planta com a água. Rio de Janeiro: EDUR. 191p.

RICHARDS RA. 2000. Selectable traits to increase crop photosynthesis and yield of grain crops. Journal of Experimental Botany 51: 447-458.

SONG Q et al. 2013. Optimal crop canopy architecture to maximize canopy photosynthetic $\mathrm{CO}_{2}$ uptake under elevated $\mathrm{CO}_{2}-\mathrm{a}$ theoretical study using a mechanistic model of canopy photosynthesis. Functional Plant Biology 40: 109-124.

SOUZA FS \& ROSOLEM CA. 2007. Rainfall intensity and mepiquat chloride persistence in cotton. Scientia Agricola 64: $125-130$.

SOUZA VQ et al. 2013. Desfolha em diferentes estádios fenológicos sobre características agronômicas em trigo. Bioscience Journal 29: 1905-1911.

TRAUTMANN RR et al. 2014. Potencial de água do solo e adubação com boro no crescimento e absorção do nutriente pela cultura da soja. Revista Brasileira de Ciência do Solo 38: 240-251.

THOLEN D et al. 2012. Opinion: Prospects for improving photosynthesis by altering leaf anatomy. Plant Science 197: $92-$ 101.

YANG XC \& HWA CM. 2008. Genetic modification of plant architecture and variety improvement in rice. Heredity 101 : 396-404.

WANG Y \& LI J. 2008. Molecular Basis of plant architecture. Annual review of plant biology 59: 253-279.

ZAGONEL J \& FERNANDES EC. 2007. Doses e épocas de aplicação de redutor de crescimento afetando cultivares de trigo em duas doses de nitrogênio. Planta Daninha 25: 331-339.

ZAGONEL $J$ et al. 2002. Efeito de regulador de crescimento na cultura de trigo submetido a diferentes doses de nitrogênio e densidades de plantas. Planta Daninha 20: 471-476. 